\title{
Comparison of pregnancy outcomes using a time- lapse monitoring system for embryo incubation versus a conventional incubator in in vitro fertilization: An age-stratification analysis
}

\author{
Pattraporn Chera-aree, Isarin Thanaboonyawat, Benjawan Thokha, Pitak Laokirkkiat
}

Infertility and Reproductive Biology Unit, Department of Obstetrics and Gynecology, Faculty of Medicine, Siriraj Hospital, Mahidol University, Bangkok, Thailand

Objective: The aim of this study was to compare the pregnancy outcomes of in vitro fertilization with embryo transfer between embryos cultured in a time-lapse monitoring system (TLS) and those cultured in a conventional incubator (Cl).

Methods: The medical records of 250 fertilized embryos from 141 patients undergoing infertility treatment with assisted reproductive technology at a tertiary hospital from June 2018 to May 2020 were reviewed. The study population was divided into TLS and Cl groups at a 1 to 1 ratio (125 embryos per group). The primary outcome was the live birth rate.

Results: The TLS group had a significantly higher clinical pregnancy rate $(46.4 \%$ vs. $27.2 \%, p=0.002)$, implantation rate $(27.1 \%$ vs. $12.0 \%$, $p=0.004)$, and live birth rate ( $32.0 \%$ vs. $18.4 \%, p=0.013)$ than the $\mathrm{Cl}$ group. Furthermore, subgroup analyses of the clinical pregnancy rate and live birth rate in the different age groups favored the TLS group. However, this difference only reached statistical significance in the live birth rate in women aged over 40 years and the clinical pregnancy rate in women aged $35-40$ years ( $p=0.048$ and $p=0.031$, respectively). The miscarriage rate, cleavage rate, and blastocyst rate were comparable.

Conclusion: TLS application improved the live birth rate, implantation rate, and clinical pregnancy rate, particularly in the advanced age group in this study, while the other reproductive outcomes were comparable. Large randomized controlled trials are needed to further explore the ramifications of these findings, especially in different age groups.

Keywords: Embryo culture techniques; Incubator; Live birth rate; Pregnancy rate; Time lapse

\section{Introduction}

With the increasing prevalence of infertility and subfertility world-

Received: September 17, 2020 • Revised: December 26, 2020 · Accepted: January 11,2021

Corresponding author: Isarin Thanaboonyawat Infertility and Reproductive Biology Unit, Department of Obstetrics and Gynecology, Faculty of Medicine, Siriraj Hospital, Mahidol University, 2 Wanglang Road, Bangkoknoi, Bangkok 10700, Thailand

Tel: +66-2-4197000 Fax: +66-2-4182662 E-mail: isarin.tha@mahidol.ac.th

This is an Open Access article distributed under the terms of the Creative Commons Attribution Non-Commercial License (http://creativecommons.org/licenses/by-nc/4.0/) which permits unrestricted non-commercial use, distribution, and reproduction in any medium, provided the original work is properly cited. wide [1,2], more people are seeking fertility treatment to achieve pregnancy. In Thailand, the prevalence of infertility is about 12\% [3]. The major causes of infertility can be divided into female factors, male factors, and unexplained infertility. Advanced age, tubal occlusion, or severe endometriosis in women, or severe male-factor infertility, may require the use of assisted reproductive technology (ART) to achieve pregnancy [4-6]. Although major advances in ART have been made since the first successful in vitro fertilization (IVF)-conceived birth in 1978, the live birth rate has remained low, at only $10 \%-30 \%$, in patients with good embryo transfer [7]. Several factors impact the success rate of ART, although these predominantly involve embryo quality and endometrial receptivity. 
Embryo quality assessment is a crucial step in ART that influences pregnancy outcomes. Many methods, including both invasive and non-invasive techniques, have been developed to evaluate embryo quality for better implantation prediction [8]. Morphological assessment in a conventional incubator $(\mathrm{Cl})$ is one of the standard noninvasive methods in this step [9]. This is a familiar method for both clinicians and embryologists to grade embryos to select the best one for transfer. However, there are several limitations of this technique [10]. First, the embryologist must remove the embryo from the incubator at least once daily for assessment. This can impact the environment surrounding the embryo and can cause deleterious effects [11]. Moreover, segmental monitoring of the embryo in $\mathrm{Cl}$ can miss some phenomena that occur during the developmental process, such as direct or reverse cleavage. These kinetic changes are associated with poor embryo quality and low implantation potential $[12,13]$. The time-lapse monitoring system (TLS), comprising an automatic device with a built-in camera, is a novel incubator that provides an undisturbed culture system and permits regular, uninterrupted monitoring of the developing embryo [14]. Therefore, TLS provides another dimension of monitoring the dynamic morphokinetic changes of embryos in addition to $\mathrm{Cl}$ [15-17]. It is able to detect kinetic changes and abnormal cleavage patterns that could be missed in the $\mathrm{Cl}$ system and enhances the consistency of embryo scoring by different embryologists [18]. In 2011, Meseguer et al. [19] conducted a retrospective study to create a model for assessing the kinetic parameters from TLS to classify embryos according to their probability of successful implantation. Several studies have shown associations of kinetic markers from TLS with pregnancy outcomes. In particular, the selection of a good-quality embryo that has been monitored in TLS for transfer enhances the implantation rate, increases the cumulative pregnancy rate, decreases early pregnancy loss, and reduces the time to pregnancy [14,19-24].

However, several confounding factors need to be clarified before concluding that this hypothesis has been confirmed, especially regarding patients' reproductive baseline and the culture environment conditions. Although several studies have provided support for the role of TLS in promoting good embryo development, others have not [14]. One randomized controlled trial that compared embryos cultured in TLS with embryos for which conventional once-daily morphologic screening was performed with additional kinetic data did not show an improvement in predicting clinical pregnancy or implantation success [17]. That study did not find an advantage of having additional morphokinetic data from an undisturbed incubation environment. Moreover, several studies have found that TLS did not contribute to improving the developmental potential of embryos, especially in patients with a favorable prognosis $[25,26]$. Consequently, to date, there is no consensus regarding the benefits of TLS over $\mathrm{Cl}[17,27]$, and the advantage of utilizing TLS over Cl to improve pregnancy outcomes has not yet been established.

In addition, the generalized application of TLS might not be cost-effective in all populations. To guide clinical practice, more high-quality evidence needs to be obtained from a larger trial or verified data from subgroup analyses specifically related to an age-adjusted population $[28,29]$. Consequently, the present study aimed to compare pregnancy outcomes between TLS and $\mathrm{Cl}$ in different age groups to determine the most appropriate indicators for identifying patients who would benefit from utilizing TLS for embryo culture and for monitoring embryo development.

\section{Methods}

This retrospective study was conducted from June 2018 to May 2020. Electronic medical records of infertile women who underwent intracytoplasmic sperm injection (ICSI) with embryo transfer at the Infertility Unit of the Department of Obstetrics and Gynecology, Faculty of Medicine Siriraj Hospital, Mahidol University were reviewed, with the data collected after Institutional Review Board approval (COA no.SI338/2018). There was no informed consent due to we conducted the study as a retrospective study. Patients aged 18-45 years undergoing autologous ICSI cycles with blastocyst transfers were included in this study. Embryos that had been rewarmed more than 4 times were excluded.

The controlled ovarian hyperstimulation $(\mathrm{COH})$ protocols of both groups were chosen through a multifactorial approach, including age, antral follicle count, basal hormone levels, cause of infertility, and history of a previous treatment cycle. Exogenous follicle-stimulating hormone with or without luteinizing hormone $(\mathrm{LH})$ was used for follicle stimulation under antagonist, agonist, or another protocol for suppressing premature LH surges. When two or more follicles reached $17 \mathrm{~mm}$ in diameter, ovum pick-up (OPU) was performed 34-36 hours later, after the induction of maturation by recombinant human chorionic gonadotropin (rhCG) or a gonadotropin-releasing hormone $(\mathrm{GnRH})$ agonist. Fertilization by ICSI was performed in all patients. The study participants were classified into two groups (TLS and $\mathrm{Cl}$ ) based on the type of incubator used. The designation of the monitoring modality was made at the discretion of the physician and embryologist.

\section{Fertilization and embryo culture}

Following follicle aspiration, oocytes were placed in $0.5 \mathrm{~mL}$ Universal IVF Medium (Origio; Cooper-Surgical, Malov, Denmark) in a fourwell dish in an MCO-230AICUVH CO 2 incubator (Panasonic, Osaka, Japan) under $37^{\circ} \mathrm{C}$ and $6 \% \mathrm{CO}_{2}$. The cumulus- oocyte complexes were soaked in a medium containing hyaluronidase (Sage, Coo- 
per-Surgical) first and then the cumulus cells were removed by mechanical pipetting 2-3 hours after OPU.

All metaphase II oocytes were fertilized using standard ICSI procedures at $\times 400$ magnification with an inverted microscope (Olympus IX71, Tokyo, Japan) 39-41 hours after rhCG or GnRH agonist administration. The injection was performed in Sydney IVF Cleavage Medium (Cook Medical, Bloomington, IN, USA) under an oil overlay. Fertilization was assessed 16-18 hours after insemination. The normally fertilized zygotes were transferred to a pre-equilibrated culture plate that had been prepared overnight within the incubator (Panasonic Healthcare, Osaka, Japan) at $37^{\circ} \mathrm{C}$ with $6 \% \mathrm{CO}_{2}$ and $5.5 \% \mathrm{O}_{2}$.

The embryos were sequentially cultured in Sydney IVF Cleavage Medium and Sydney IVF Blastocyst Medium (Cook Medical), using either TLS (Embryoscope time-lapse system; Vitrolife, Gothenburg, Sweden) or Cl under an oil (Sage) overlay. For standard cultivation or $\mathrm{Cl}$, a 35-mm dish was prepared with $10 \mu \mathrm{L}$ of Sydney IVF Cleavage Medium. The media was changed to Sydney IVF Blastocyst Medium at day 3 under $37^{\circ} \mathrm{C}$ with a $5 \% \mathrm{CO}_{2}, 5 \% \mathrm{O}_{2}$, and $90 \% \mathrm{~N}_{2}$ atmosphere in a mini-incubator (Planer Benchtop Incubator BT37, Cooper-Surgical). The embryos were scored according to the blastomere numbers, size, and amount of fragmentation daily in the morning following the consensus checkpoints for standard embryo assessment [10]. For the time-lapse imaging system (EmbryoScope time-lapse system, Vitrolife), a 12-well Embryoslide culture dish was prepared with $25 \mu \mathrm{L}$ of Sydney IVF Cleavage Medium and the medium was refreshed after 48 hours with Sydney IVF Blastocyst Medium under the same temperature and gas concentration settings as $\mathrm{Cl}$. Embryo images were captured from seven focal planes every 10 minutes. The embryos in the TLS group were selected for transfer based on morphology, the presence or absence of abnormal cleavage, and morphokinetic parameters, including time to 2 cells (t2), 4 cells (t4), 5 cells (t5), and the interval between 3 and 4 cells (s2).

\section{Embryo transfer method}

Fresh or frozen embryo transfer (FET) was selected by the physician based on the number of oocytes retrieved, endometrial morphology, and pre-implantation genetic test planning. The blastocysts were transferred 5 days after oocyte retrieval in fresh embryo transfer. Vaginal progesterone for luteal support was administered on the day of OPU or 1 day after. For FET, vaginal progesterone was added 5-6 days before the blastocysts were transferred after artificial endometrial preparation by estradiol. Embryo transfer was performed under transabdominal ultrasound guidance in all cases.

\section{Outcome measurements}

The patients' baseline characteristics, including age, body mass index (BMI), and type and cause of infertility, were collected. Cycle characteristics, including the stimulation protocol, duration of stimulation, number of oocytes retrieved, maturation rate, and fertilization rates, were also recorded. The live birth rate, which was the primary outcome, was defined as delivery of the fetus beyond 28 weeks of gestational age. The secondary outcomes were the implantation rate, clinical pregnancy rate, cleavage rate, blastocyst rate, miscarriage rate, and age-stratification of the live birth rate and clinical pregnancy rate. The implantation rate was defined as the number of gestational sacs identified by ultrasonography divided by the number of embryos transferred, while the clinical pregnancy rate was defined as the presence of gestational sacs on ultrasonography.

The number of oocytes retrieved was defined as the total number of oocytes that were collected, while the maturation rate was calculated as the number of metaphase II oocytes divided by the total number of oocytes retrieved. The fertilization rate was defined as the number of two pronuclear (2PN) zygotes divided by the total number of inseminated mature oocytes. The total number of day $3 \mathrm{em}$ bryos divided by the number of 2PN zygotes gave the cleavage rate, and the total number of blastocysts divided by the number of 2PN zygotes was defined as the blastocyst rate.

\section{Statistical analysis}

Based on a previous study, the live birth rates of embryo transfers from the use of TLS and $\mathrm{Cl}$ were $45 \%$ and $28 \%$, respectively [4]. Considering a power of $80 \%$ for the study and a type 1 error of $5 \%$, our study required 125 embryo transfers per group. All statistical analyses were performed using PASW ver. 18.0 (SPSS Inc., Chicago, IL, USA). The baseline characteristics and cycle outcomes in the TLS and $\mathrm{Cl}$ groups were defined as the number (\%) and mean \pm standard deviation when normally distributed. Categorical outcomes between the TLS and $\mathrm{Cl}$ groups were compared using analysis of variance, the Fisher exact test, or the chi-square test as appropriate. The independent Student $t$-test or Mann-Whitney U-test was used for a comparative analysis of continuous variables. Subgroup analysis was used to evaluate the primary outcome in the three different age groups. The baseline parameters that were significantly different between the TSL and $\mathrm{Cl}$ groups and those that may impact the primary endpoint were considered to be possible confounding factors and were analyzed by logistic regression. All the significance tests were two-tailed, with an a level of 0.05 . A $p$-value of less than 0.05 was considered to indicate statistical significance.

\section{Results}

In total, 141 patients underwent ICSI cycles with 250 embryo transfers (125 in the TLS group and 125 in the Cl group) and were enrolled in the study. The baseline characteristics of the patients are 
Table 1. Baseline characteristics of participants with embryos cultured in the TLS and Cl groups

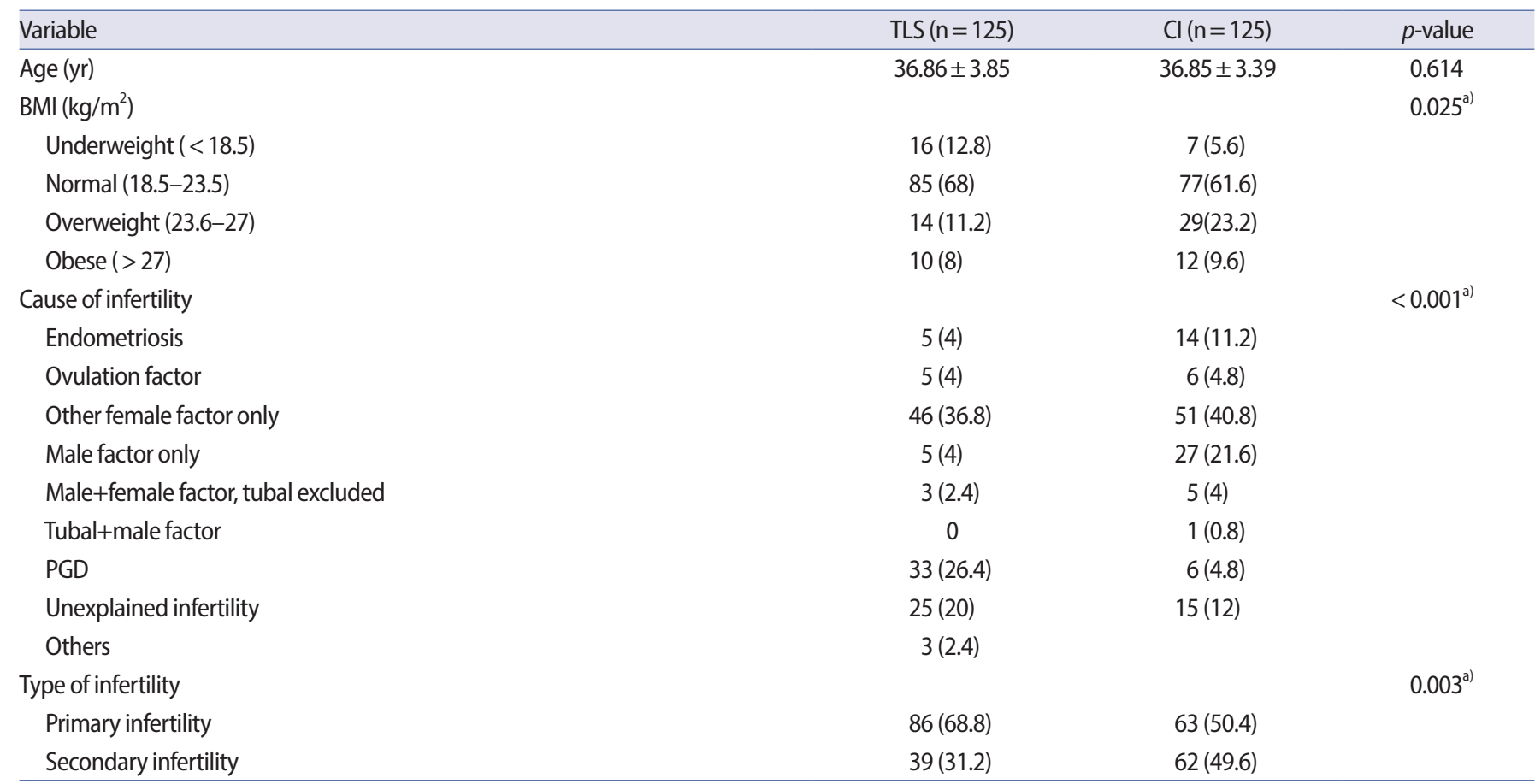

Values are presented as mean \pm standard deviation or number (\%).

TLS, time-lapse monitoring system; $\mathrm{Cl}$, conventional incubator; $\mathrm{BMI}$, body mass index; $\mathrm{PGD}$, preimplantation genetic diagnosis. ${ }^{\text {a) }} p<0.05$.

demonstrated in Table 1. The mean age in both groups was 36.8 years. The participants in both groups predominantly had a BMI in the normal range. Female factors were the major cause of infertility in both groups. The second most common cause of infertility in the Cl group was male factors (21.6\%), while ICSI for preimplantation genetic diagnosis (PGD) was the second most common reason for ART in the TLS group (26.4\%). Most patients had primary infertility in both groups. The $\mathrm{COH}$ cycle characteristics are presented in Table 2. The mean duration of stimulation, mean number of retrieved oocytes, and the stimulation protocol used were comparable in both groups. The maturation rate and fertilization rate were also similar. However, there was a higher number of participants in the TLS group than in the $\mathrm{Cl}$ group who underwent fresh embryo transfer $(p=0.002)$. More preimplantation genetic screening (PGS) or PGD was performed in the TLS group than in the $\mathrm{Cl}$ group $(p<0.001)$. The reproductive outcomes are shown in Table 3. TLS demonstrated a higher live birth rate $(32.0 \%$ vs. $18.4 \%, p=0.013)$, implantation rate $(27.1 \%$ vs. $12.0 \%, p=0.004)$ and clinical pregnancy rate $(46.4 \%$ vs. $27.2 \%, p=0.002)$ than the $\mathrm{Cl}$ group. The miscarriage rates in the TLS and $\mathrm{Cl}$ groups were $6.4 \%$ and $5.6 \%$, respectively $(p=0.79)$. There were 10 patients (8\%) in the TLS group and four patients (3.2\%) in the $\mathrm{Cl}$ group who developed chemical pregnancy followed by a spontaneous decrease in beta-human chorionic gonadotropin. The cleavage rate was over $90 \%$ and the blastocyst rate was over $70 \%$ in both groups. The results from the subgroup analysis of the live birth rate, clinical pregnancy rate, and miscarriage rate stratified by age groups are listed in Table 4. There were 65 embryo transfer cycles for patients aged $<35$ years (group A), 141 embryo transfer cycles for patients aged 35-40 years (group B), and 44 embryo transfer cycles for patients aged $>40$ years old (group C). Statistical significance was retained regarding the live birth rate in group C ( $34.8 \%$ vs. 9.5\%, $p=0.048)$ and the clinical pregnancy rate for group B $(45.7 \%$ vs. $28.2 \%, p=0.031)$. The live birth rates in the TLS and $\mathrm{Cl}$ groups were $40.6 \%$ and $21.2 \%(p=0.090), 27.1 \%$ and $19.7 \%(p=0.298)$, and $34.8 \%$ and $9.5 \%(p=0.048)$ in groups $A, B$, and $C$, respectively, as shown in Figure 1. The miscarriage rates in the three age groups were not significantly different. The live birth rate adjusted by confounding factors (PGS and type of embryo transfer) is presented in Table 5 and logistic regression analysis of these parameters is displayed in Table 6. There was no significant difference in the live birth rate between PGS and non-PGS, between fresh and FET embryo transfer cycles, or according to $\mathrm{BMI}$ range. These parameters did not affect the favorable outcomes of TLS over $\mathrm{Cl}$, although this did not reach statistical significance. 
Table 2. Controlled ovarian hyperstimulation cycle characteristics in the TLS and Cl groups

\begin{tabular}{|c|c|c|c|}
\hline Characteristics & TLS $(n=125)$ & $\mathrm{Cl}(\mathrm{n}=125)$ & $p$-value \\
\hline Duration of stimulation (day) & $9.4 \pm 1.3$ & $9.4 \pm 1.2$ & $0.950^{\mathrm{a})}$ \\
\hline No. of oocytes retrieved & $15.64 \pm 13.50$ & $13.8 \pm 6.95$ & $0.966^{\mathrm{a}}$ \\
\hline Maturation rate (\%) & $79.22 \pm 16.74$ & $78.42 \pm 16.33$ & $0.510^{\mathrm{a})}$ \\
\hline Fertilization rate (\%) & $80.78 \pm 17.51$ & $81.69 \pm 16.32$ & $0.880^{\mathrm{a})}$ \\
\hline \multicolumn{4}{|l|}{ Stimulation protocol } \\
\hline Antagonist & $108(86.4)$ & $114(91.2)$ & $0.504^{b)}$ \\
\hline Long agonist & $4(3.2)$ & $3(2.4)$ & \\
\hline Other & $7(5.6)$ & $6(4.8)$ & \\
\hline \multicolumn{4}{|l|}{ Type of embryo transfer } \\
\hline Fresh & $32(25.6)$ & $13(10.4)$ & $0.002^{\mathrm{b})}$ \\
\hline FET & $93(74.4)$ & $112(89.6)$ & \\
\hline PGS & $31(24.8)$ & $9(7.2)$ & \\
\hline PGD & $29(23.2)$ & $6(4.8)$ & \\
\hline
\end{tabular}

Values are presented as mean \pm standard deviation or number (\%).

TLS, time-lapse monitoring system; Cl, conventional incubator; FET, frozen embryo transfer; PGS, preimplantation genetic screening; PGD, preimplantation genetic diagnosis.

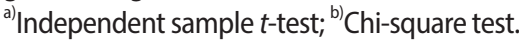

Table 3. Reproductive outcomes of transfer cycles with embryos cultured in the TLS and Cl groups

\begin{tabular}{|c|c|c|c|}
\hline Variable & $\operatorname{TLS}(n=125)$ & $\mathrm{Cl}(\mathrm{n}=125)$ & $p$-value \\
\hline Live birth rate & $40(32.0)$ & $23(18.4)$ & $0.013^{\mathrm{a})}$ \\
\hline Clinical pregnancy rate & $58(46.4)$ & $34(27.2)$ & $0.002^{\mathrm{a})}$ \\
\hline Implantation rate (\%) & 27.1 & 12.0 & $0.004^{b)}$ \\
\hline Chemical pregnancy rate & $10(8)$ & $4(3.2)$ & $0.099^{\mathrm{a})}$ \\
\hline Miscarriage rate & $8(6.4)$ & $7(5.6)$ & $0.790^{\mathrm{a})}$ \\
\hline Cleavage rate (\%) & $93.26 \pm 14.88$ & $93.54 \pm 12.66$ & 0.370 \\
\hline
\end{tabular}

Values are presented as number (\%) or mean \pm standard deviation.

TLS, time-lapse monitoring system; $\mathrm{Cl}$, conventional incubator.

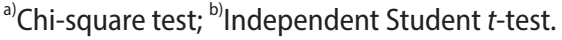

\section{Discussion}

One of the key factors for a successful pregnancy from ART is good embryo quality. An embryo monitoring incubator is essential equipment that also impacts the embryo quality. Good embryo grading for embryo transfer depends on the optimal culture environment and having precise morphological information. A standard incubator (or $\mathrm{Cl}$ ) provides a stable culture environment, including the temperature, $\mathrm{pH}$, humidity, and gas concentration [23]. Usually, when a $\mathrm{Cl}$ is used, embryo morphology is assessed under an inverted microscope by an embryologist once daily. This process has the advantage that information can be obtained on the embryo development and some dysmorphic events can be observed, but at the same time, the use of a Cl can lead to a disturbance of the culture environment [23]. Moreover, some significant kinetic changes in the embryo cannot be tracked without continuous observation [10,30,31]. The development of TLS, a novel automated embryo incubator, can overcome the limitations of a $\mathrm{Cl}$ by allowing continuous monitoring of embryo development in favorable constant culture conditions.

Our study clearly showed better reproductive outcomes, including a higher live birth rate, implantation rate, and clinical pregnancy rate with a lower miscarriage rate, in the TLS group than in the $\mathrm{Cl}$ group. The purification of gas by constant recirculation through an active filter in TLS may facilitate embryo development. Moreover, the tem- 
Table 4. Reproductive outcomes in subgroup analyses of TLS and $\mathrm{Cl}$ based on patients' age groups

\begin{tabular}{lcccc}
\hline Variable & TLS (\%) & $\mathrm{Cl}(\%)$ & Difference (\%) & $p$-value \\
\hline Live birth rate & & & & \\
$\quad<35 \mathrm{yr}$ & 40.6 & 21.2 & 19.5 & 0.090 \\
$35-40 \mathrm{yr}$ & 27.1 & 19.7 & 7.4 & 0.298 \\
$>40 \mathrm{yr}$ & 34.8 & 9.5 & 25.3 & 0.048 \\
$\quad$ All & 32.0 & 18.4 & 13.6 & 0.013 \\
Clinical pregnancy rate & & & & \\
$\quad<35 \mathrm{yr}$ & 50.0 & 30.3 & 19.7 & 0.105 \\
$35-40 \mathrm{yr}$ & 45.7 & 28.2 & 17.5 & 0.031 \\
$>40 \mathrm{yr}$ & 45.3 & 19.0 & 24.5 & 0.082 \\
All & 46.4 & 27.2 & 19.2 & 0.002 \\
Miscarriage rate & & & & \\
$<35 \mathrm{yr}$ & 0 & 9.1 & 9.1 & 0.083 \\
$35-40 \mathrm{yr}$ & 10.0 & 4.2 & 5.8 & 0.182 \\
$>40 \mathrm{yr}$ & 4.3 & 4.8 & 0.5 & 0.947 \\
All & 6.4 & 5.6 & 0.8 & 0.790 \\
\hline
\end{tabular}

Age: $<35(n=65), 35-40(n=141),>40(n=44)$, all $(n=250)$.

TLS, time-lapse monitoring system; $\mathrm{Cl}$, conventional incubator.

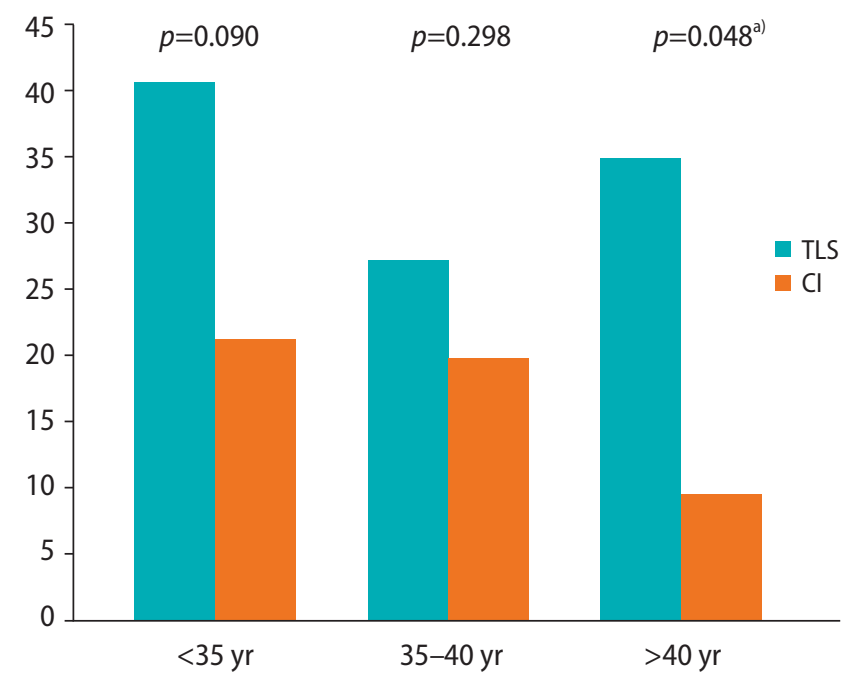

Figure 1. Live birth rate in time-lapse monitoring system (TLS) and conventional incubator $(\mathrm{Cl})$ stratified by age groups. ${ }^{\text {a) }} p<0.05$.

Table 5. Live birth rate adjusted by non-PGS versus PGS and fresh versus FET in the TLS and $\mathrm{Cl}$ groups

\begin{tabular}{lccc}
\hline Variable & TLS $(\%, n=125)$ & $\mathrm{Cl}(\%, \mathrm{n}=125)$ & $p$-value \\
\hline Non-PGS & & & \\
Fresh $(\mathrm{n}=44)$ & 38.7 & 23.1 & 0.318 \\
FET $(\mathrm{n}=166)$ & 23.8 & 16.5 & 0.247 \\
PGS & & & \\
Fresh $(\mathrm{n}=1)$ & 100 & 0 & NA \\
FET $(\mathrm{n}=39)$ & 40 & 33.3 & 0.718 \\
\hline
\end{tabular}

PGS, preimplantation genetic screening; FET, frozen embryo transfer; TLS, time-lapse monitoring system; $\mathrm{Cl}$, conventional incubator; NA, not available. perature and gas concentrations in TLS recover more quickly to optimal conditions after doors are opened than in $\mathrm{Cl}$ [23]. In the age subgroup analysis, the live birth rate was higher in participants whose embryos were monitored in TLS in all subgroups, although, the result only reached statistical significance in participants aged $>40$ years. This may be a result of age-related changes in embryo quality. Given the high prevalence of good embryo quality in younger patients, this suggests that TLS might not be necessary to discriminate embryo quality for better pregnancy outcomes in this age group. On the contrary, in advanced-age patients with a high prevalence of poor-quality embryos, specifically with aneuploidy [32], TLS assists in identifying embryos at high risk of complex aneuploidy by allowing the monitoring of some critical morphokinetic parameters, specifically the time point of the second cell division ( $\mathrm{t} 3$ ) and the time interval between 2 and 5 cells ( $\mathrm{t} 5-\mathrm{t} 2$ ) [33]. Therefore, it can play a crucial role in improving pregnancy outcomes by enabling the clinician to select and discard low-implantation-potential embryos. Moreover, the cleavage pattern has been suggested to be associated with embryo quality. Direct and reverse cleavage, for example, are phenomena associated with poor embryo quality [13]. In the Cl group, embryos with direct or reverse cleavage may be lost to tracking and may be mislabeled as good embryos instead [12,34,35].

Not only the cleavage sequence, but also atypical embryo phenotypes, including abnormal syngamy, abnormal first cytokinesis, abnormal cleavage, and chaotic cleavage associated with poor implantation potential, can be detected by TLS [13,34]. Both the morphology and development series were considered during embryo selection in the TLS group. As a result, only the embryos that followed the general timeline were considered the best for transfer. In other words, TLS can facilitate the prioritization of embryo transfer.

Many previous studies have mentioned the benefits of TLS for pregnancy outcomes; however, there is still no consensus yet [36]. The study of Siristatidis et al. [4], similar to ours, showed a higher live birth rate in the TLS monitoring group, especially in participants aged $>40$ years old. They proposed that having more details for the embryological assessment and the stable culture conditions of TLS contributed to these outcomes. In the present study, as has been reported in other studies, TLS allowed the continuous monitoring of embryo development in an uninterrupted environment and enabled the detection of morphokinetic events that can help predict implantation potential and pregnancy outcomes [12,19,23,30,37-39]. In contrast to the present study, however, other reports did not find any significant improvement in reproductive outcomes even when TLS was applied [14,17,25]. In 2014, Polanski et al. [40] reported two randomized controlled trials comparing efficacy and safety between TLS and $\mathrm{Cl}$. Their study did not find a substantial benefit of TLS over Cl, although they also stated that the use of this method did not pose a 
Table 6. Evaluation of possible confounding factors on the live birth rate by logistic regression analysis (including the type of incubator, PGS vs. non- PGS, fresh vs. FET, and BMI)

\begin{tabular}{lccc}
\hline Variable & B & OR (95\% confidence interval) \\
\hline Method & - & 1 & $p$-value \\
Cl & 0.478 & $1.61(0.85-3.06)$ & 0.142 \\
TLS & & 1 & 0.090 \\
PGS and type of embryo transfer & - & $0.527(0.25-1.31)$ & 0.100 \\
Non-PGS, fresh & -0.641 & $1.127(0.45-2.86)$ & 0.802 \\
Non-PGS, FET & 0.119 & 1 & 0.978 \\
PGS, FET & & $0.798(0.29-2.18)$ & 0.660 \\
BMI (kg/m ${ }^{2}$ ) & - & $0.836(0.25-2.77)$ \\
Underweight & -0.226 & $0.794(0.19-3.27)$ \\
Normal & -0.179 & 0.770 \\
Overweight & -0.231 & 0.749 \\
Obese & & & \\
\hline
\end{tabular}

PGS, preimplantation genetic screening; FET, frozen embryo transfer; BMI, body mass index; OR, odds ratio; $\mathrm{Cl}$, conventional incubator; TLS, time-lapse monitoring system.

risk or cause any harm. In addition to the study of Polanski et al. [40], other systematic reviews have revealed insufficient data to support the routine use of TLS for embryo selection, and TLS has typically been considered an experimental intervention since then [27,41,42]. It should be noted that these studies were performed on participants with different individual and cycle-level characteristics from our study. In particular, studies that showed a significant improvement in the pregnancy rate were mainly undertaken with cleavage transfer $[4,37]$. In light of the scarcity of high-quality literature, several trials have been conducted to determine the exact roles for which TLS is most suitable for embryo monitoring. A significant advantage of TLS was shown in a recent meta-analysis [24], which demonstrated that the application of TLS was associated with an increased live birth rate, higher ongoing pregnancy rate, and lower early pregnancy loss, although the quality of the study was still not good enough to draw a firm conclusion.

At our center, we usually prefer to place embryos that need PGD or PGS in TLS for blastocyst culture. Thus, significantly more PGD or PGS tests were performed in the TLS group. For this reason, we utilized a logistic regression model to analyze all the significant confounding factors that may impact pregnancy outcomes from the different types of incubators. Although several studies have demonstrated a significant improvement in the ongoing pregnancy rate and implantation rate in embryos with PGS testing [43-45], the present study did not find any statistically significant differences in the live birth rate according to whether PGS was performed in both groups. As with PGS, fresh or FET and BMI were not identified as significant factors contributing to improvements in the live birth rate in the logistic regression analysis. After accounting for all the confounding factors, the effect of TLS over Cl had an odds ratio of 1.61 (95\% confidence interval, 0.85-3.06), although it did not reach statistical significance. Our study presented the clinical significance of TLS for improving the live birth rate compared to $\mathrm{Cl}$. It should be noted that the live birth rate achieved with TLS was higher than that for $\mathrm{Cl}$ in all subgroups, although the population was too small to reach statistical significance.

Our study was conducted in a population with a variety of baseline characteristics. Only patients who underwent blastocyst transfer were recruited and embryos that were cultured for at least 300 runs every 10 minutes in the TLS group were selected. In this context, we assumed that the time period in the TLS group was adequate to reflect the environmental effect of this incubator system. Moreover, we analyzed the live birth rate to determine the final outcome of pregnancy, which might give information on the cost-effectiveness of applying TLS to improve pregnancy outcomes. Nevertheless, the retrospective nature of the study could be a limitation of our study. The causes of infertility were different between the groups. Although the most common causes of infertility were the same, more participants who underwent PGD were present in the TLS group. Some patients need PGD to prevent pregnancy with a diseased child, not to treat infertility, such as high-risk couples with alpha- and beta-thalassemia. Thus, the higher number of other causes in the $\mathrm{Cl}$ group may have led to poorer reproductive outcomes in this group. Other confounding factors and selection bias should also be considered when interpreting the results. Moreover, the different culture conditions in the two types of incubators might have affected the embryo quality in different ways.

In conclusion, TLS application improved the live birth rate, implantation rate, and clinical pregnancy rate in patients with an older maternal age. The differences in outcomes between the two types of incubators studied (TLS and $\mathrm{Cl}$ ) tended to become more evident as 
age increased. A study with a larger sample size is needed to find strong evidence to specifically support the advantage of TLS in different age groups.

\section{Conflict of interest}

No potential conflict of interest relevant to this article was reported.

\section{Acknowledgments}

The authors would like to thank Dr. Suphanut Danphichitsuk, a sixth-year medical student, who helped to extract the data from electronic medical records, and Assist. Prof. Dr. Chulaluk Komoltri, a clinical epidemiologist at the Faculty of Medicine Siriraj Hospital, Mahidol University, for assistance with the statistical analysis.

\section{ORCID}

Pattraporn Chera-aree https://orcid.org/0000-0001-8373-9537 Isarin Thanaboonyawat https://orcid.org/0000-0002-3912-1382 Benjawan Thokha https://orcid.org/0000-0002-0988-1479 Pitak Laokirkkiat https://orcid.org/0000-0001-5277-1526

\section{Author contributions}

Conceptualization: PC, IT. Data curation: PC, BT. Formal analysis, Methodology, \& Investigation: PC, IT. Writing-original draft: PC. Writing-review \& editing: PC, IT, PL.

\section{References}

1. Zhang H, Wang S, Zhang S, Wang T, Deng X. Increasing trend of prevalence of infertility in Beijing. Chin Med J (Engl) 2014;127: 691-5.

2. Dick ML, Bain CJ, Purdie DM, Siskind V, Molloy D, Green AC. Self-reported difficulty in conceiving as a measure of infertility. Hum Reprod 2003;18:2711-7.

3. Tuntiseranee P, Olsen J, Chongsuvivatwong V, Limbutara S. Fecundity in Thai and European regions: results based on waiting time to pregnancy. Hum Reprod 1998;13:471-7.

4. Siristatidis C, Komitopoulou MA, Makris A, Sialakouma A, Botzaki M, Mastorakos G, et al. Morphokinetic parameters of early embryo development via time lapse monitoring and their effect on embryo selection and ICSI outcomes: a prospective cohort study. J Assist Reprod Genet 2015;32:563-70.

5. Kuivasaari P, Hippelainen M, Anttila M, Heinonen S. Effect of en- dometriosis on IVF/ICSI outcome: stage III/IV endometriosis worsens cumulative pregnancy and live-born rates. Hum Reprod 2005;20:3130-5.

6. Diedrich K, Felberbaum R, Kupker W, al-Hasani S. New approaches to male infertility: IVF and microinjection. Int J Androl 1995;18 Suppl 2:78-80.

7. Martin JR, Bromer JG, Sakkas D, Patrizio P. Live babies born per oocyte retrieved in a subpopulation of oocyte donors with repetitive reproductive success. Fertil Steril 2010;94:2064-8.

8. Nel-Themaat L, Nagy ZP. A review of the promises and pitfalls of oocyte and embryo metabolomics. Placenta 2011;32 Suppl 3: S257-63.

9. Cummins JM, Breen TM, Harrison KL, Shaw JM, Wilson LM, Hennessey JF. A formula for scoring human embryo growth rates in in vitro fertilization: its value in predicting pregnancy and in comparison with visual estimates of embryo quality. J In Vitro Fert Embryo Transf 1986;3:284-95.

10. Alpha Scientists in Reproductive Medicine and ESHRE Special Interest Group of Embryology. The Istanbul consensus workshop on embryo assessment: proceedings of an expert meeting. Hum Reprod 2011;26:1270-83.

11. Fujiwara M, Takahashi K, Izuno M, Duan YR, Kazono M, Kimura F, et al. Effect of micro-environment maintenance on embryo culture after in-vitro fertilization: comparison of top-load mini incubator and conventional front-load incubator. J Assist Reprod Genet 2007:24:5-9.

12. Rubio I, Kuhlmann R, Agerholm I, Kirk J, Herrero J, Escriba MJ, et al. Limited implantation success of direct-cleaved human zygotes: a time-lapse study. Fertil Steril 2012;98:1458-63.

13. Liu Y, Chapple V, Roberts P, Matson P. Prevalence, consequence, and significance of reverse cleavage by human embryos viewed with the use of the Embryoscope time-lapse video system. Fertil Steril 2014;102:1295-300.e2.

14. Cruz M, Gadea B, Garrido N, Pedersen KS, Martinez M, Perez-Cano I, et al. Embryo quality, blastocyst and ongoing pregnancy rates in oocyte donation patients whose embryos were monitored by time-lapse imaging. J Assist Reprod Genet 2011;28:569-73.

15. Herrero J, Meseguer M. Selection of high potential embryos using time-lapse imaging: the era of morphokinetics. Fertil Steril 2013, 99:1030-4.

16. Desai N, Ploskonka S, Goodman LR, Austin C, Goldberg J, Falcone T. Analysis of embryo morphokinetics, multinucleation and cleavage anomalies using continuous time-lapse monitoring in blastocyst transfer cycles. Reprod Biol Endocrinol 2014;12:54.

17. Goodman LR, Goldberg J, Falcone T, Austin C, Desai N. Does the addition of time-lapse morphokinetics in the selection of embryos for transfer improve pregnancy rates? A randomized controlled 
trial. Fertil Steril 2016;105:275-85.e10.

18. Sundvall L, Ingerslev HJ, Breth Knudsen U, Kirkegaard K. Inter- and intra-observer variability of time-lapse annotations. Hum Reprod 2013;28:3215-21.

19. Meseguer M, Herrero J, Tejera A, Hilligsoe KM, Ramsing NB, Remohi J. The use of morphokinetics as a predictor of embryo implantation. Hum Reprod 2011;26:2658-71.

20. Zhang JQ, Li XL, Peng Y, Guo X, Heng BC, Tong GQ. Reduction in exposure of human embryos outside the incubator enhances embryo quality and blastulation rate. Reprod Biomed Online 2010;20:510-5.

21. Castello D, Motato Y, Basile N, Remohi J, Espejo-Catena M, Meseguer M. How much have we learned from time-lapse in clinical IVF? Mol Hum Reprod 2016;22:719-27.

22. Ebner T, Oppelt P, Radler E, Allerstorfer C, Habelsberger A, Mayer $\mathrm{RB}$, et al. Morphokinetics of vitrified and warmed blastocysts predicts implantation potential. J Assist Reprod Genet 2017;34:23944.

23. Meseguer M, Rubio I, Cruz M, Basile N, Marcos J, Requena A. Embryo incubation and selection in a time-lapse monitoring system improves pregnancy outcome compared with a standard incubator: a retrospective cohort study. Fertil Steril 2012;98:1481-9.e10.

24. Pribenszky C, Nilselid AM, Montag M. Time-lapse culture with morphokinetic embryo selection improves pregnancy and live birth chances and reduces early pregnancy loss: a meta-analysis. Reprod Biomed Online 2017;35:511-20.

25. Kirkegaard K, Kesmodel US, Hindkjær JJ, Ingerslev HJ. Time-lapse parameters as predictors of blastocyst development and pregnancy outcome in embryos from good prognosis patients: a prospective cohort study. Hum Reprod 2013;28:2643-51.

26. Kirkegaard K, Hindkjaer JJ, Grondahl ML, Kesmodel US, Ingerslev $\mathrm{HJ}$. A randomized clinical trial comparing embryo culture in a conventional incubator with a time-lapse incubator. J Assist Reprod Genet 2012;29:565-72.

27. Racowsky C, Kovacs P, Martins WP. A critical appraisal of time-lapse imaging for embryo selection: where are we and where do we need to go? J Assist Reprod Genet 2015;32:1025-30.

28. Montag M. Morphokinetics and embryo aneuploidy: has time come or not yet? Reprod Biomed Online 2013;26:528-30.

29. Ottolini C, Rienzi L, Capalbo A. A cautionary note against embryo aneuploidy risk assessment using time-lapse imaging. Reprod Biomed Online 2014;28:273-5.

30. Lemmen JG, Agerholm I, Ziebe S. Kinetic markers of human embryo quality using time-lapse recordings of IVF/ICSI-fertilized oocytes. Reprod Biomed Online 2008;17:385-91.

31. Sciorio R, Thong JK, Pickering SJ. Comparison of the development of human embryos cultured in either an EmbryoScope or bench- top incubator. J Assist Reprod Genet 2018;35:515-22.

32. Scheffer JB, Scheffer BB, de Carvalho RF, Rodrigues J, Grynberg M, Mendez Lozano DH. Age as a predictor of embryo quality regardless of the quantitative ovarian response. Int J Fertil Steril 2017; 11:40-6.

33. Del Carmen Nogales M, Bronet F, Basile N, Martinez EM, Linan A, Rodrigo $L$, et al. Type of chromosome abnormality affects embryo morphology dynamics. Fertil Steril 2017;107:229-35.e2.

34. Athayde Wirka K, Chen AA, Conaghan J, Ivani K, Gvakharia M, Behr $B$, et al. Atypical embryo phenotypes identified by time-lapse microscopy: high prevalence and association with embryo development. Fertil Steril 2014;101:1637-48.e1-5.

35. Zhan Q, Ye Z, Clarke R, Rosenwaks Z, Zaninovic N. Direct unequal cleavages: embryo developmental competence, genetic constitution and clinical outcome. PLoS One 2016;11:e0166398.

36. Kirkegaard K, Ahlstrom A, Ingerslev HJ, Hardarson T. Choosing the best embryo by time lapse versus standard morphology. Fertil Steril 2015;103:323-32.

37. Rubio I, Galan A, Larreategui Z, Ayerdi F, Bellver J, Herrero J, et al. Clinical validation of embryo culture and selection by morphokinetic analysis: a randomized, controlled trial of the EmbryoScope. Fertil Steril 2014;102:1287-94.e5.

38. Wong CC, Loewke KE, Bossert NL, Behr B, De Jonge CJ, Baer TM, et al. Non-invasive imaging of human embryos before embryonic genome activation predicts development to the blastocyst stage. Nat Biotechnol 2010;28:1115-21.

39. Conaghan J, Chen AA, Willman SP, Ivani K, Chenette PE, Boostanfar $\mathrm{R}$, et al. Improving embryo selection using a computer-automated time-lapse image analysis test plus day 3 morphology: results from a prospective multicenter trial. Fertil Steril 2013;100: 412-9.e5.

40. Polanski LT, Coelho Neto MA, Nastri CO, Navarro PA, Ferriani RA, Raine-Fenning $\mathrm{N}$, et al. Time-lapse embryo imaging for improving reproductive outcomes: systematic review and meta-analysis. UItrasound Obstet Gynecol 2014;44:394-401.

41. Armstrong S, Vail A, Mastenbroek S, Jordan V, Farquhar C. Timelapse in the IVF-lab: how should we assess potential benefit? Hum Reprod 2015;30:3-8.

42. Armstrong S, Arroll N, Cree LM, Jordan V, Farquhar C. Time-lapse systems for embryo incubation and assessment in assisted reproduction. Cochrane Database Syst Rev 2015;(2):CD011320.

43. Scott RT Jr, Upham KM, Forman EJ, Hong KH, Scott KL, Taylor D, et al. Blastocyst biopsy with comprehensive chromosome screening and fresh embryo transfer significantly increases in vitro fertilization implantation and delivery rates: a randomized controlled trial. Fertil Steril 2013;100:697-703.

44. Forman EJ, Hong KH, Ferry KM, Tao X, Taylor D, Levy B, et al. In vitro 
fertilization with single euploid blastocyst transfer: a randomized controlled trial. Fertil Steril 2013;100:100-7.e1.

45. Yang Z, Liu J, Collins GS, Salem SA, Liu X, Lyle SS, et al. Selection of single blastocysts for fresh transfer via standard morphology as- sessment alone and with array CGH for good prognosis IVF patients: results from a randomized pilot study. Mol Cytogenet 2012;5:24. 